\title{
Layered-resolved autofluorescence imaging of photoreceptors using two-photon excitation
}

\author{
Ling-Ling Zhao, Jun-Le Qu', Dan-Ni Chen, Han-Ben Niu
}

Key Laboratory of Optoelectronic Devices and Systems of Ministry of Education, Institute of Optoelectronics, Shenzhen University, Shenzhen, China.

Email: jlqu@szu.edu.cn

Received 21 April 2009; revised 15 May 2009; accepted 19 May 2009.

\begin{abstract}
In this paper, we present our investigation on the morphological and autofluorescence characteristics of the cones and rods using twophoton excitation with a femtosecond Ti: sapphire laser. The results show that the microstructures of the photoreceptor layers can be visualized at submicron level without any staining or slicing. The morphology and spatial distribution of the cones and rods can be resolved by autofluorescence imaging. The autofluorescence in the photoreceptor outer segments is much stronger than other layers, but susceptible to light-induced damage.
\end{abstract}

Keywords: Two-Photon Excitation; Photoreceptor; Retina; Autofluorescence

\section{INTRODUCTION}

There are two basic types of photoreceptors, rods and cones, which are of different shapes and involved in different visual functions. Rods are highly sensitive to the weak and faint light, which are used for vision under dark-dim conditions at night. Cones are the basis of our color perceptions since they have different wavelength sensitivity and the consequent pathways of connectivity to the brain. The dysfunction of photoreceptors is one of most important factors of ocular fundus diseases, such as retinitis pigmentosa (RP), age-related macular degeneration (AMD), glaucoma, and so forth. [1,2] Retinitis pigmentosa is the leading cause of inherited blindness, which is characterized by progressive loss of visual function related to death of rods then cones, and leads to the breakdown of the photoreceptor outer segment disc membranes. Patients with retinitis pigmentosa (RP) show various symptoms. The onset is often gradual and insidious, and many patients fail to recognize the manifestations of this condition until it has progressed significantly. When patients do report symptoms, they commonly include difficulty with night vision (nyctalo- pia) as well as loss of peripheral vision. In addition, age-related macular degeneration (AMD) is a slowly progressive disease that is related to the abnormal accumulation of lipofuscin, which is located in the retinal pigment epithelium (RPE) cells and is a byproduct of incomplete digestion of photoreceptor outer segment disc membranes. Generally, the people who suffer from AMD have an early abnormal condition and experience minor visual loss. For many of these people, macular degeneration will not progress to a more serious condition. But for the others, macular degeneration may lead to severe loss of visual acuity (or centralvision). [3]

Fortunately, with the advent of near infrared femtosecond laser, multiphoton excitation fluorescence microscopy, which is based on simultaneous absorption of two or more photons, has become a novel and powerful tool in biomedical imaging. [4] It enables the possibilities to investigate the autofluoresecence and spatial distribution of photoreceptors at early stage of ocular diseases, since it can increase the penetrability through the thick biological tissue and provide high-resolution imaging of endogenous fluorophores in biological tissue. [5,6] And also, Multiphoton microscopy can facilitate the simultaneous excitation of different endogenous fluorophores in biological specimens at submicron level, which benefits to the identification and evaluation of the morphological and autofluorescence characteristics of the photoreceptors. Several endogenous fluorophores exist in the photoreceptors, e.g., all-trans-retinol, NAD (P) H, A2-PE, FAD etc, which are all related to the metabolism and visual cycle. [7,8,9,10] Therefore, multiphoton microscopy can be used to investigate the autofluorescence characteristics of photoreceptors.

In this study, layered-resolved autofluorescence imaging using two-photon excitation has been performed, which can provide not only structural, but also functional information about the different layers of photoreceptors, particularly photoreceptor inner and outer segments which are vital functional layers in the photoreceptors and significant for the early diagnosis of the retinal diseases. 


\section{MATERIALS AND METHODS}

\subsection{Materials}

Fresh porcine eyes are supplied by the local slaughterhouse and extracted immediately after slaughtering within one hour. The eyeballs are transported to the lab in PBS (PH 7.16)/normal saline solution and kept in a freezing box $\left(0-4^{\circ} \mathrm{C}\right)$. The eyes are kept in the PBS (PH 7.16)/normal saline solution for less than 30 minutes before experiment. A $6 \mathrm{~mm}$-diameter retina-RPE-choroidsclera complex near the macula (both the rods and cones are in existence) is trephined and the neurosensory retina is peeled off gingerly. The side that connects with the RPE cells is kept down to contact the slip of a specially designed chamber. All the specimens are freshly prepared without fixing, slicing and labeling.

\subsection{Methods}

The experiment is performed on an inverted confocal laser scanning microscope (Leica TCS SP2). A modelocked femtosecond Ti: sapphire laser (Coherent Mira $900 \mathrm{~F}$ ) is coupled to the microscope for two-photon excitation of the specimen. The Ti: Sapphire laser produces ultrashort laser pulses with tunable wavelength from $700 \mathrm{~nm}$ to $980 \mathrm{~nm}$, a pulse width of about $120 \mathrm{fs}$ and a repetition rate of $76 \mathrm{MHz}$. The excitation wavelength in this experiment is $800 \mathrm{~nm}$. A $63 \times / \mathrm{NA} 1.32$ oil-immersion objective is used to focus the excitation light onto the specimen and collect the autofluorescence from the photoreceptors.

\section{RESULTS AND DISCUSSIONS}

Both the rod and cone photoreceptors are consisted of four parts, i.e., the outer segment, the inner segment, the nuclear region and the synaptic terminal. The outer segment is filled with stacks of membranes containing the visual pigment molecules such as rhodopsins. The inner segment contains mitochondria, ribosomes and membranes where opsin molecules are assembled and passed to the outer segment discs. The nuclear region contains the nucleus of the photoreceptor cell and the synaptic terminal is responsible for the neurotransmission to second order neurons.

Usually rod photoreceptors are slim rod-shaped structures with their inner and outer segments filling the area between the larger cones in the subretinal space and stretching to the pigment epithelium cells. Rod cell bodies make up the remainder of the outer nuclear layer below the cone cell bodies. Cone photoreceptors, on the other hand, are robust conical-shaped structures that have their cell bodies situated in a single row right below the outer limiting membrane and their inner and outer segments protruding into the subretinal space towards the pigment epithelium. Apical processes from the pigment epithelium envelope the outer segments of both rods and cones.
In our study, we obtain the image of rod and cone photoreceptors nicely aligned along vertical and horizontal directions using two-photon excitation fluorescence microscopy and we also identify the fluorophores in photoreceptor outer segments by analyzing the spectrum, intensity, distribution and components of autofluorescence [11]. Figure 1 shows the cross-sectional autofluorescence image of photoreceptors, in which individual cones and rods are clearly resolved. The XZ image of the different photoreceptor layer structures are distinguished accurately with submicron resolution. The autofluorescence in the photoreceptor outer segments is much stronger than other layers, since the outer segment is abundant in many fluorophores, such as all-transretinol, A2-PE, FAD, etc.

The XY image of the photoreceptor outer segment is shown in Figure 2. Because the photoreceptor outer segment is connected with the RPE cells, when the photoreceptor outer segments is separated from the

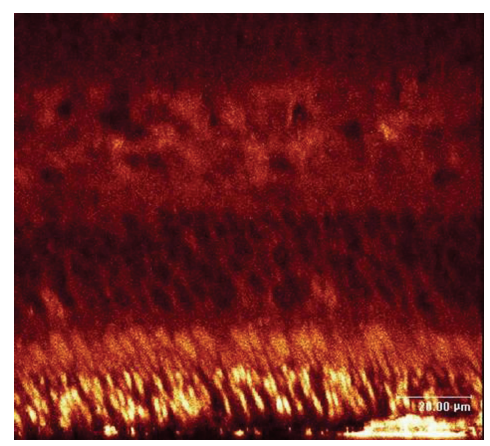

Figure 1. Cross-sectional autofluorescence image of photoreceptors excited by twophoton excitation. $\left(\lambda_{\text {Ti: Sapphire }}=800 \mathrm{~nm}\right)$.

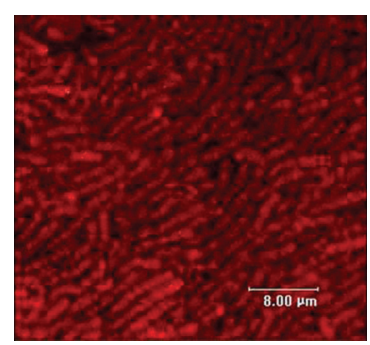

(a)

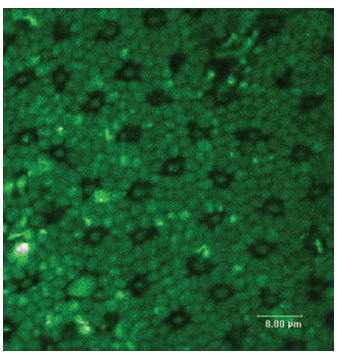

(c)

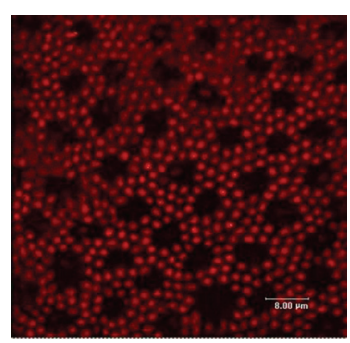

(b)

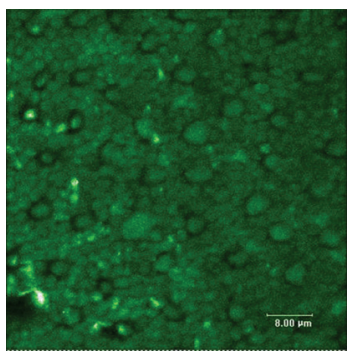

(d)
Figure 2. Autofluorescence image of photoreceptor out segment excited by two-photon excitation. $\left(\lambda_{\text {Ti: Sapphire }}=800 \mathrm{~nm}\right)$. 
RPE cells, the outer segment at top may be disarranged, but the pillar-like shape can be observed (Figure 2(a)). When focusing the imaging plane slightly into the specimen, we can see the rod is aligned annularly, round in shape and $2 \mu \mathrm{m}$ in diameter (Figure 2(b)) and the center is dark. With focusing the objective further into the specimen, the autofluorescence of cone outer segment, which locates in the center, can be detected, and the size of the cones is becoming larger and larger (Figure 2(c) and Figure 2(d)), whereas the autofluorescence of rod outer segment weakens.

Figure 3 shows the autofluorescence image of photoreceptor inner segment. The bigger cell is corresponding to the cone inner segment whose diameter is around 6 $\mu \mathrm{m}$. Rod inner segment is around the cone and the diameter is about $2 \mu \mathrm{m}$. Figure 4 is the autofluorescence image of photoreceptor nuclear region, which contains the nucleus of the photoreceptor cell.

\section{CONCLUSIONS}

Our results demonstrate, for the first time, that morphological and autofluorescence characteristics of different layers of photoreceptors can be identified by autofluorescence imaging using two-photon excitation. Since the cross section of two photon excitation is much smaller than that of single photon excitation, and two photon

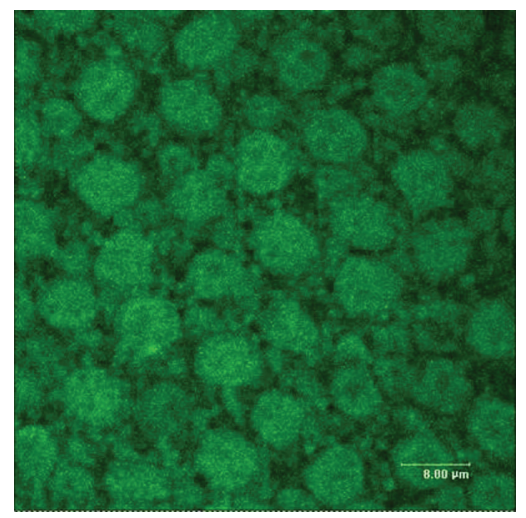

Figure 3. Autofluorescence image of photoreceptor inner segment.

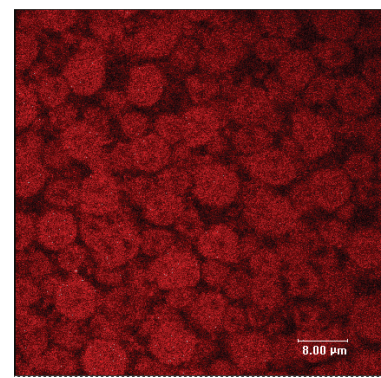

(a)

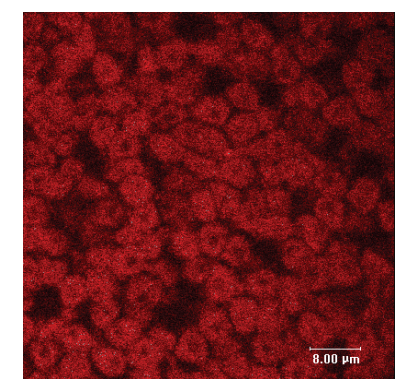

(b)
Figure 4. Autofluorescence image of cone nuclear region (a) and rod nuclear region (b), excited by wo-photon excitation $\left(\lambda_{\text {Ti: Sapphire }}=800 \mathrm{~nm}\right)$. excitation is quadratically dependent on the intensity, it is susceptible to photondamage at the focal point. In our work, the structure and properties of procine eyes are very similar to human eyes, so the laser power on the sample is limited to the order of $\mathrm{mW}(3-4 \mathrm{~mW})$, which accords with ANSI about laser safety criteria for human eye. Moreover, this in vitro autofluorescence imaging of photoreceptors can give much detailed structural and functional information at high spatial resolution, which can help to more clearly understand the in vivo ocular fundus autofluorescence images obtained by confocal scanning laser ophthalmoscopy. And also, two photon excitation is IR illumination, which can penetrate the anterior segment of eyes, such as cornea and lens, and get the whole autofluorescence characteristics of the living retina, and eliminate the autofluorescence interference of the anterior segment of eyes by tightly focus without confocal pinhole. Thus, in vitro autofluorescence imaging of photoreceptors using two photon excitation can provide a clue and develop a two-photon laser scanning ophthalmoscope for in vivo living retina imaging. Although much research remains to be done, it appears that this technique has great potential to bridge the gap between clinical examination and invasive biopsy and thus facilitate the early detection and diagnosis of ocular diseases. The distribution and intensity of autofluorescence may provide an insight into the sequence of events that leads to retinal damage and may help elucidate pathological mechanisms.

\section{ACKNOWLEDGMENTS}

This work is supported by the National Natural Science Foundation of China (NSFC) under contract No. 60627003 and No. 60408011, and is also supported in part by Guangdong Natural Science Foundation grant No. 5010500 .

\section{REFERENCES}

[1] Jiangang, G. and Kyeongmi, C., (2002) PNAS, 99(8), 5698.

[2] Kayatz, P., Thumann, G., Luther, T. T., et al., (2001) Invest. Ophthalmol. Vis. Sci., 42, 241.

[3] Hopkins, J., Walsh, A. and Chakravarthy, U., (2006) Invest. Ophthalmol. Vis. Sci., 47, 2269.

[4] Brakenhoff, G. J., Squier, J., Norris, T. and Blito, A. C., (1996) J. Microsc., 181, 253.

[5] Denk, W., Strickler, J. H., (1990) Science, 248, 73.

[6] Han, M. and Giese, G., (2007) Journal of Biomedical Optics, 12(2), 024012.

[7] Chunhe, C., (2005) Biophysical Journal, 88, 2278.

[8] Kim, S. R. and Nakanishi, K., (2006) Experimental Eye Research, 82, 828.

[9] Laura, L. E. and John, S. D., (2004) Photochemistry and Photobiology, 79(2), 127.

[10] Schweitzer, D., Schenke, S., and Hammer, M., (2007) Microscopy Research and Technique, 70, 410.

[11] Zhao, L., Qu J., and Niu, H., (2007) Proc. SPIE, 6826, 682614 . 